\title{
Precision Characteristics of the Differential Radar Reflectivity Meter
}

\author{
Evgenii V. Masalov and Nikolay N. Krivin* \\ Tomsk State University \\ of Control Systems and Radioelectronics \\ 40 Lenin, Tomsk, 634050, Russia
}

Received 09.08.2017, received in revised form 23.10.2017, accepted 21.01.2018

The paper studies the questions relating to the analysis of the accuracy characteristics of the differential radar reflection meter as applied to radar probing tasks of the medium filled with hydrometeors. It is well known the efficiency of the differential radar reflectivity method has limited by its strong dependence on orientation angle between medium basis and radar polarization basis. The estimation of radar reflectivity measurement error is determined to such limitations. A new approach to the estimation of precision characteristics of the radar reflectivity meter has presented. The method had based on the use of dependence of the scattering matrix weight coefficient on the medium factors such as differential phase shift and differential attenuation. The particularity of proposed approach is calculation relations destined to the radar reflectivity variability determination and error measurement estimation. The calculation of the differential radar reflectivity and its error measurement estimation has presented. The given result is analyzed.

Keywords: polarization, differential phase shift, differential attenuation, differential radar reflectivity, radar reflectivity measurement error, scattering matrix.

Citation: Masalov E.V., Krivin N.N. Precision characteristics of the differential radar reflectivity meter, J. Sib. Fed. Univ. Eng. technol., 2019, 12(1), 97-105. DOI: 10.17516/1999-494X-0028.

(C) Siberian Federal University. All rights reserved

This work is licensed under a Creative Commons Attribution-NonCommercial 4.0 International License (CC BY-NC 4.0).

* Corresponding author E-mail address: e-v-masalov@yandex.ru, freeman46@yandex.ru 


\title{
Точностные характеристики
}

\section{радиолокационного измерителя дифференциальной радиолокационной отражаемости}

\author{
Е.В. Масалов, Н.Н. Кривин \\ Томский государственный университет \\ систем управления и радиоэлектроники \\ Россия, 634050, Томск, пр. Ленина, 40
}

В работе рассмотрены вопросы, связанные с анализом точностных характеристик радиолокачионного измерителя дифференциильной радиолокационной отражаемости применительно $\kappa$ задачам радиолокачионного зондирования среды, заполненной гидрометеорами. С учетом того, что к числу факторов, ограничивающих эффективность метода дифференциальной радиолокационной отражаемости, относится его выраженная зависимость от угла ориентации собственного поляризационного базиса среды, заполненной гидрометеорами, относительно измерительного базиса, проводится определение погрешности в оиенке величины данного параметра. Предложен подход для оценки точностных характеристик радиолокационного измерителя, основанный на использовании функииональной зависимости весового коэффициента матрицы рассеяния от дифференциального ослабления и дифференциального фазового сдвига. Особенностью предлагаемого подхода являются расчетные соотношения как для определения самой изменчивости величины дифференциальной радиолокачионной отражаемости, так и для оченки погрешности ее измерения. Приводятся результаты расчетов величины дифференциальной радиолокационной отражаемости и погрешности в ее оценке. Полученные результаты анализируются.

Ключевые слова: поляризачия, дифференциильный фазовый сдвиг, дифференциальное ослабление, дифференииальная радиолокаиионная отражаемость, погрешность измерения дифференциальной радиолокачионной отражсаемости, матрица рассеяния.

\section{Введение}

Поляризационный радиолокатор, измеряющий величину дифференциальной радиолокационной отражаемости (ДРЛО) $\mathrm{Z}_{\mathrm{DR}}(\mathrm{z})$, реализует один из простейших возможных алгоритмов использования поляризационной структуры радиолокационного сигнала, основанный на поочередном излучении сигнала с вертикальной линейной поляризацией и приеме отраженного сигнала на ту же антенну, а также излучении сигнала с горизонтальной линейной поляризацией и приеме отраженного сигнала на ту же антенну.

В силу сложности процессов трансформации поляризационной структуры зондирующего сигнала, проходящего через среду гидрометеоров, особое значение приобретает интерпретация результатов измерений, а также точность, с которой они сделаны [1-4].

\section{Постановка задачи}

Существенное воздействие дифференциального фазового сдвига $\Delta \Phi$ и дифференциального ослабления $\Delta \alpha$ на поляризационную структуру зондирующего радиолокационного сигнала является причиной неадекватности результатов оценки интенсивности осадков и алгоритмов 
распознавания опасных зон метеообразований на основе поляризационных измерений с применением двух ортогональных линейных поляризаций [1].

В связи с этим возникает необходимость получения оценки изменчивости дифференциальной радиолокационной отражаемости и величины погрешности $\Delta \mathrm{Z}_{\mathrm{DR}}(\mathrm{z})$ в оценке величины ДРЛО, возникающей при воздействии указанных выше параметров среды: $\Delta \alpha, \Delta \Phi$,

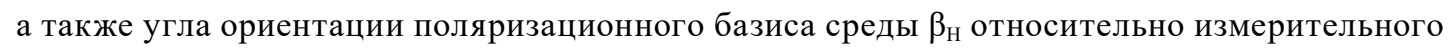
базиса.

\section{Методика решения}

Величина дифференциальной радиолокационной отражаемости $Z_{\mathrm{DR}}(\mathrm{z})$ на выходе приемника с логарифмической характеристикой может быть определена в виде [5]

$$
\mathrm{Z}_{\mathrm{DR}}(\mathrm{z})=20 \cdot \lg \left(\left|\dot{\mathrm{E}}_{\mathrm{XX}}(\mathrm{z})\right| /\left|\dot{\mathrm{E}}_{\mathrm{YY}}(\mathrm{z})\right|\right)
$$

где $\left|\dot{\mathrm{E}}_{\mathrm{XX}}(\mathrm{z})\right|$ - амплитуда сигнала, излученного с горизонтальной поляризацией и принятого той же антенной; $\left|\dot{\mathrm{E}}_{\mathrm{YY}}(\mathrm{z})\right|$ - амплитуда сигнала излученного с вертикальной поляризацией и принятого той же антенной.

Поскольку основной характеристикой метеообразования, дающей возможность получить оценку $\mathrm{Z}_{\mathrm{DR}}(\mathrm{z})$, является матрица рассеяния, то для решения поставленной задачи необходимо использовать такое выражение этой матрицы, параметры которого учитывали бы воздействие указанных выше факторов.

В качестве такой матрицы рассеяния может быть использовано выражение, аналогичное приведенному в работе [6], которое с учетом того, что в равномерных дождях большая полуось эллипсоида вращения, аппроксимирующего форму капель, ориентирована в горизонтальной плоскости [6, 7], может быть представлено в виде

$$
\mathbf{S}=\left[\begin{array}{cc}
\cos \beta_{\mathrm{H}} & -\sin \beta_{\mathrm{H}} \\
\sin \beta_{\mathrm{H}} & \cos \beta_{\mathrm{H}}
\end{array}\right]\left[\begin{array}{cc}
1 & 0 \\
0 & 10^{-0,05 \Delta \alpha \mathrm{z}} \cdot \mathrm{e}^{-\mathrm{j} \Delta \Phi \mathrm{z}}
\end{array}\right]\left[\begin{array}{cc}
\cos \beta_{\mathrm{H}} & \sin \beta_{\mathrm{H}} \\
-\sin \beta_{\mathrm{H}} & \cos \beta_{\mathrm{H}}
\end{array}\right]
$$

где $\mathrm{z}$ - длина трассы распространения зондирующего сигнала.

После перемножения матриц в (2) и переходя к тригонометрическим функциям двойных углов, получим:

$$
\begin{gathered}
\mathbf{S}=0,5\left(1+10^{-0,05 \Delta \alpha \mathrm{z}} \cdot \mathrm{e}^{-\mathrm{j} \Delta \Phi \mathrm{z}}\right) \cdot\left[\begin{array}{ll}
1 & 0 \\
0 & 1
\end{array}\right]+ \\
+0,5\left(1-10^{-0,05 \Delta \alpha \mathrm{z}} \cdot \mathrm{e}^{-\mathrm{j} \Delta \Phi \mathrm{z}}\right) \cdot\left[\begin{array}{cc}
\cos 2 \beta_{\mathrm{H}} & \sin 2 \beta_{\mathrm{H}} \\
\sin 2 \beta_{\mathrm{H}} & -\cos 2 \beta_{\mathrm{H}}
\end{array}\right] .
\end{gathered}
$$

Вынося множитель $0,5\left(1+10^{-0,05 \Delta \alpha z} \cdot \mathrm{e}^{-\mathrm{j} \Delta \Phi \mathrm{z}}\right)$ за скобки, получим окончательное выражение для матрицы рассеяния в виде

$$
\begin{gathered}
\mathbf{S}=0,5\left(1+10^{-0,05 \Delta \alpha z} \cdot \mathrm{e}^{-\mathrm{j} \Delta \Phi \mathrm{z}}\right) \cdot \\
\cdot\left\{\left[\begin{array}{ll}
1 & 0 \\
0 & 1
\end{array}\right]+\dot{\mathrm{M}}(\mathrm{z}) \cdot\left[\begin{array}{cc}
\cos 2 \beta_{\mathrm{H}} & \sin 2 \beta_{\mathrm{H}} \\
\sin 2 \beta_{\mathrm{H}} & -\cos 2 \beta_{\mathrm{H}}
\end{array}\right]\right\} \\
-99-
\end{gathered}
$$


где весовой коэффициент $\dot{M}(\mathrm{z})$ равен

$$
\begin{gathered}
\dot{\mathrm{M}}(\mathrm{z})=\frac{1-10^{-0,05 \Delta \alpha \mathrm{z}} \cdot \mathrm{e}^{-\mathrm{j} \Delta \Phi \mathrm{z}}}{1+10^{-0,05 \Delta \alpha \mathrm{z}} \cdot \mathrm{e}^{-\mathrm{j} \Delta \Phi \mathrm{z}}}= \\
=\frac{\sqrt{1-2 \cdot 10^{-0,05 \Delta \alpha \mathrm{z}} \cdot \cos \Delta \Phi \mathrm{z}+10^{-0,1 \Delta \alpha \mathrm{z}}}}{\sqrt{1+2 \cdot 10^{-0,05 \Delta \alpha \mathrm{z}} \cdot \cos \Delta \Phi \mathrm{z}+10^{-0,1 \Delta \alpha \mathrm{z}}}} \times \\
\times \mathrm{e}^{\mathrm{j}\left(\delta_{1}-\delta_{2}\right)}=|\dot{\mathrm{M}}(\mathrm{z})| \mathrm{e}^{\mathrm{j} \delta},
\end{gathered}
$$

где $\delta_{1}=\operatorname{arctg}\left(\frac{10^{-0,05 \Delta \alpha \mathrm{z}} \sin \Delta \Phi \mathrm{z}}{1-10^{-0,05 \Delta \alpha \mathrm{z}} \cos \Delta \Phi \mathrm{z}}\right) ; \delta_{2}=\operatorname{arctg}\left(\frac{-10^{-0,05 \Delta \alpha \mathrm{z}} \sin \Delta \Phi \mathrm{z}}{1+10^{-0,05 \Delta \alpha \mathrm{z}} \cos \Delta \Phi \mathrm{z}}\right)$;

$\delta=\delta_{1}-\delta_{2}=\operatorname{arctg}\left(\frac{2 \cdot 10^{-0,05 \Delta \alpha \mathrm{z}} \sin \Delta \Phi \mathrm{z}}{1-10^{-0,1 \Delta \alpha \mathrm{z}}}\right)$.

Чтобы найти амплитуды $\left|\dot{\mathrm{E}}_{\mathrm{XX}}(\mathrm{z})\right|$ и $\left|\dot{\mathrm{E}}_{\mathrm{YY}}(\mathrm{z})\right|$, подставим матрицу рассеяния (5) в следующие выражения

$$
\begin{aligned}
& \dot{\mathrm{E}}_{\mathrm{XX}}(\mathrm{z})=\left[\begin{array}{ll}
1 & 0 \\
0 & 0
\end{array}\right][\mathbf{S}]\left[\begin{array}{l}
1 \\
0
\end{array}\right]=\left|\dot{\mathrm{E}}_{\mathrm{XX}}(\mathrm{z})\right| \mathrm{e}^{\mathrm{j}\left(\delta_{2}+\varphi_{1}\right)}, \\
& \dot{\mathrm{E}}_{\mathrm{YY}}(\mathrm{z})=\left[\begin{array}{ll}
0 & 0 \\
0 & 1
\end{array}\right][\mathbf{S}]\left[\begin{array}{l}
0 \\
1
\end{array}\right]=\left|\dot{\mathrm{E}}_{\mathrm{YY}}(\mathrm{z})\right| \mathrm{e}^{\mathrm{j}\left(\delta_{2}+\varphi_{2}\right)},
\end{aligned}
$$

где множители, стоящие слева перед матрицей $\mathbf{S}$, представляют собой матрицы Джонса прямоугольных волноводов, обеспечивающих прием сигнала с горизонтальной поляризацией для $\left|\dot{E}_{X X}(\mathrm{z})\right|$ и с вертикальной поляризацией для $\left|\dot{\mathrm{E}}_{\mathrm{YY}}(\mathrm{z})\right|$ соответственно; множители, стоящие справа от матрицы $\mathbf{S},-$ вектора Джонса электромагнитной волны, излученной с горизонтальной и вертикальной поляризациями соответственно.

Осуществляя перемножение в (6) и (7), получим окончательно:

$$
\begin{aligned}
& \left|\dot{\mathrm{E}}_{\mathrm{XX}}(\mathrm{z})\right|=0,5 \sqrt{\mathrm{B}} \sqrt{1+\left(\frac{\mathrm{A}}{\mathrm{B}}\right) \cos ^{2} 2 \beta_{\mathrm{H}}+2 \sqrt{\frac{\mathrm{A}}{\mathrm{B}}} \cdot \cos \delta \cdot \cos 2 \beta_{\mathrm{H}}} \\
& \left|\dot{\mathrm{E}}_{\mathrm{YY}}(\mathrm{z})\right|=0,5 \sqrt{\mathrm{B}} \sqrt{1+\left(\frac{\mathrm{A}}{\mathrm{B}}\right) \cos ^{2} 2 \beta_{\mathrm{H}}-2 \sqrt{\frac{\mathrm{A}}{\mathrm{B}}} \cdot \cos \delta \cdot \cos 2 \beta_{\mathrm{H}}} \\
& \varphi_{1}=\operatorname{arctg}\left(\frac{\sqrt{\mathrm{A}} \cdot \cos 2 \beta_{\mathrm{H}} \cdot \sin \delta}{\sqrt{\mathrm{B}}+\sqrt{\mathrm{A}} \cdot \cos 2 \beta_{\mathrm{H}} \cdot \cos \delta}\right) \\
& \varphi_{2}=\operatorname{arctg}\left(\frac{-\sqrt{\mathrm{A}} \cdot \cos 2 \beta_{\mathrm{H}} \cdot \sin \delta}{\sqrt{\mathrm{B}}-\sqrt{\mathrm{A}} \cdot \cos 2 \beta_{\mathrm{H}} \cdot \cos \delta}\right)
\end{aligned}
$$

где $\mathrm{A}=1-2 \cdot 10^{-0,05 \Delta \alpha \mathrm{z}} \cdot \cos \Delta \Phi \mathrm{z}+10^{-0,1 \Delta \alpha \mathrm{z}} ; \mathrm{B}=1+2 \cdot 10^{-0,05 \Delta \alpha \mathrm{z}} \cdot \cos \Delta \Phi \mathrm{z}+10^{-0,1 \Delta \alpha \mathrm{z}}$.

Анализ выражений (6), (7) показывает, что величина дифференциальной радиолокационной отражаемости $Z_{\mathrm{DR}}(\mathrm{z})$, рассчитанная по формуле (1), несет истинную информацию о дифференциальных факторах среды только в том случае, если $\beta_{\mathrm{H}}=0$, т.е. когда ориентация измерительного базиса совпадает с ориентацией собственного базиса метеообъекта. Во всех остальных случаях величина ДРЛО зависит как от величины $\beta_{\mathrm{H}}$, так и от дифференциальных факторов метеосреды.

На рис. 1-3 изображены расчетные зависимости $\mathrm{Z}_{\mathrm{DR}}(\mathrm{z})$. 


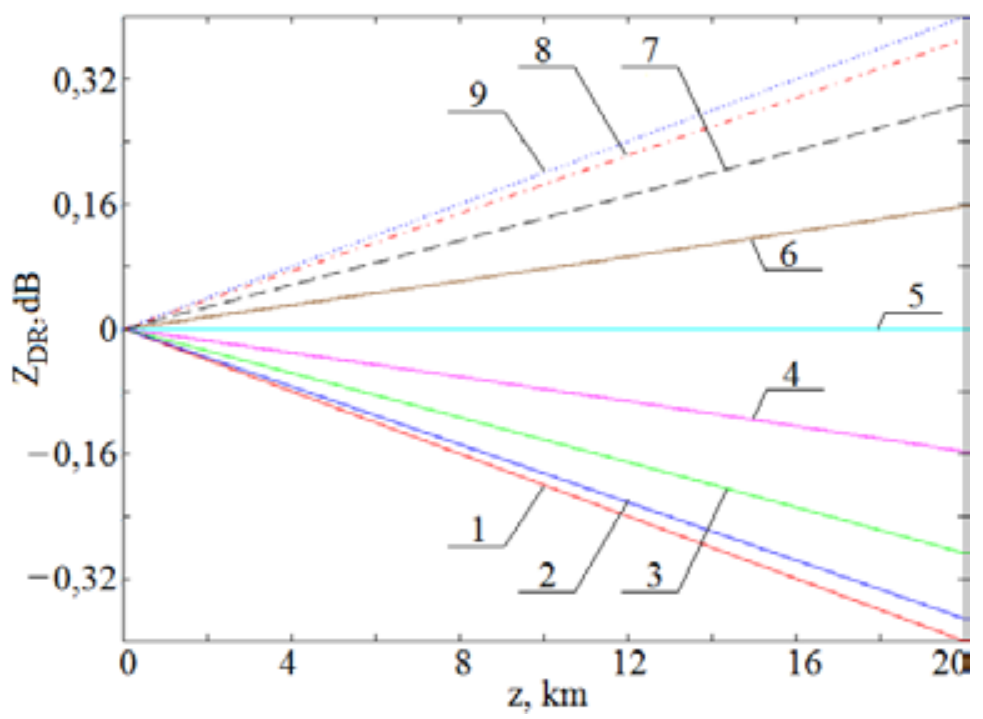

Рис. 1. Зависимость $Z_{\mathrm{DR}}(\mathrm{z})$ при $\mathrm{R}=12,5$ мм/ч при значениях $\beta_{\mathrm{H}}: 1-0^{\mathrm{o}} ; 2-11,25^{\circ} ; 3-22,5^{\circ} ; 4-33,75^{\circ} ; 5-45^{\circ}$; $6-56,25^{\circ} ; 7-67,5^{\circ} ; 8-78,75^{\circ} ; 9-90^{\circ}$

Fig. $1 . \mathrm{Z}_{\mathrm{DR}}(\mathrm{z})$ as a function of observation distance $z$ for the rain intensity $\mathrm{R}=12,5 \mathrm{~mm} / \mathrm{h}$ and polarization basis orientation angle $\beta_{\mathrm{H}}$ (in degrees): $1-0 ; 2-11,25 ; 3-22,5 ; 4-33,75 ; 5-45 ; 6-56,25 ; 7-67,5 ; 8-78,75 ; 9-90$

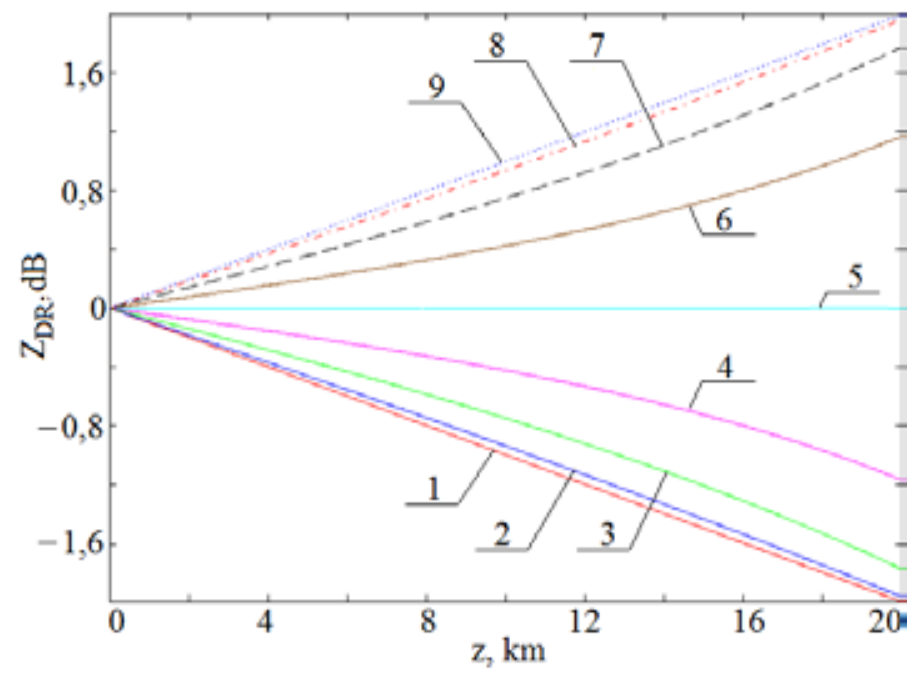

Рис. 2. Зависимость $Z_{\mathrm{DR}}(\mathrm{z})$ при $\mathrm{R}=50$ мм/ч при значениях $\beta_{\mathrm{H}}: 1-0^{\mathrm{o}} ; 2-11,25^{\circ} ; 3-22,5^{\circ} ; 4-33,75^{\circ} ; 5-45^{\circ}$; $6-56,25^{\circ} ; 7-67,5^{\circ} ; 8-78,75^{\circ} ; 9-90^{\circ}$

Fig. 2. $Z_{\mathrm{DR}}(\mathrm{z})$ as a function of observation distance $z$ for the rain intensity $\mathrm{R}=50 \mathrm{~mm} / \mathrm{h}$ and polarization basis orientation angle $\beta_{\mathrm{H}}$ (in degrees): $1-0 ; 2-11,25 ; 3-22,5 ; 4-33,75 ; 5-45 ; 6-56,25 ; 7-67,5 ; 8-78,75 ; 9-90$

В расчетах использовались частотные зависимости измеренных значений дифференциального ослабления $\Delta \alpha$ и дифференциального фазового сдвига $\Delta \Phi$, приведенные в [8]. В случае сигналов трехсантиметрового диапазона для различных значений интенсивностей осадков $\mathrm{R}$ эти величины составили:

- при $\mathrm{R}=12,5$ мм/ч: $\Delta \alpha=0,02$ Дб/км; $\Delta \Phi=1$ град/км; 


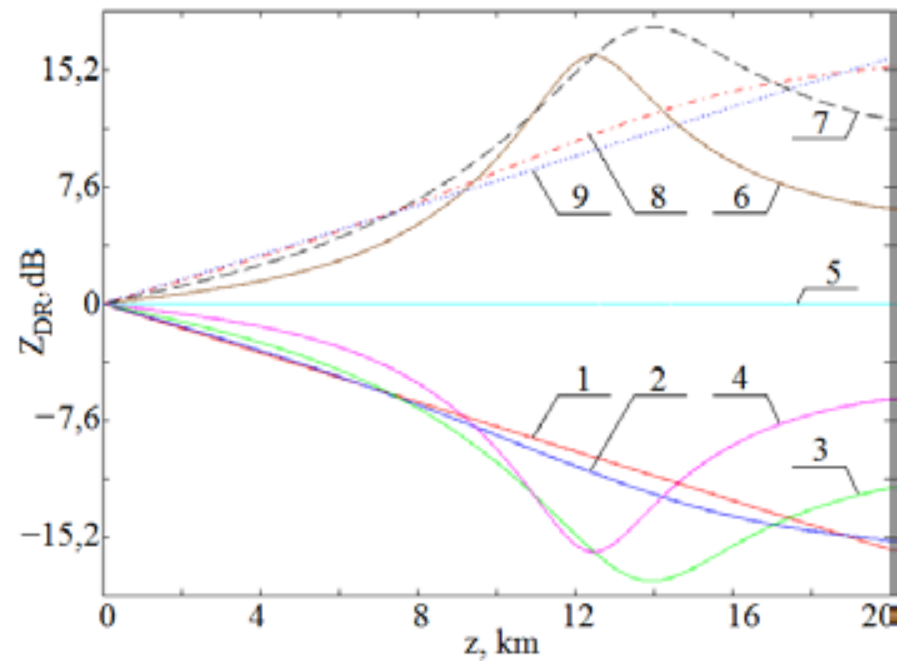

Рис. 3. Зависимость $\mathrm{Z}_{\mathrm{DR}}(\mathrm{z})$ при $\mathrm{R}=150$ мм/ч при значениях $\beta_{\mathrm{H}}: 1-0^{\circ} ; 2-11,25^{\circ} ; 3-22,5^{\circ} ; 4-33,75^{\circ} ; 5-45^{\circ}$; $6-56,25^{\circ} ; 7-67,5^{\circ} ; 8-78,75^{\circ} ; 9-90^{\circ}$

Fig. 3. $\mathrm{Z}_{\mathrm{DR}}(\mathrm{z})$ as a function of observation distance $z$ for the rain intensity $\mathrm{R}=150 \mathrm{~mm} / \mathrm{h}$ and polarization basis orientation angle $\beta_{\mathrm{H}}$ (in degrees): $1-0 ; 2-11,25 ; 3-22,5 ; 4-33,75 ; 5-45 ; 6-56,25 ; 7-67,5 ; 8-78,75 ; 9-90$

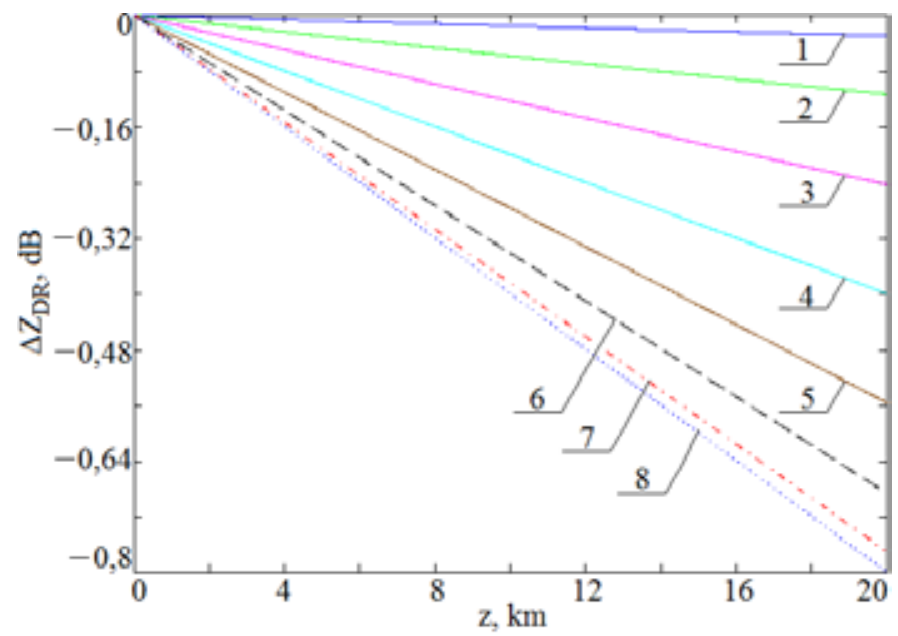

Рис. 4. $\Delta \mathrm{Z}_{\mathrm{DR}}$ при $\mathrm{R}=12,5$ мм/ч при значениях $\beta_{\mathrm{Hi}}: 1-11,25^{\circ} ; 2-22,5^{\circ} ; 3-33,75^{\circ} ; 4-45^{\circ} ; 5-56,25^{\circ} ; 6-67,5^{\circ}$; $7-78,75^{\circ} ; 8-90^{\circ}$

Fig. 4. Differential radar reflectivity measurement error $\Delta \mathrm{Z}_{\mathrm{DR}}$ for the rain intensity $\mathrm{R}=12,5 \mathrm{~mm} / \mathrm{h}$ and polarization basis orientation angle $\beta_{\mathrm{Hi}}$ (in degrees): $1-0 ; 2-11,25 ; 3-22,5 ; 4-33,75 ; 5-45 ; 6-56,25 ; 7-67,5$; $8-78,75 ; 9-90$

- при $\mathrm{R}=50$ мм/ч: $\Delta \alpha=0,1$ Дб/км; $\Delta \Phi=4$ град/км;

- при $\mathrm{R}=150$ мм/ч: $\Delta \alpha=0,8$ Дб/км; $\Delta \Phi=14$ град/км.

Расчеты выполнялись для сигналов с линейной поляризацией излучаемой волны при следующих дискретных значениях ориентации собственного базиса среды $\beta_{\mathrm{H}}$ относительно измерительного базиса: $0^{\circ} ; 11,25^{\circ} ; 22,5^{\circ} ; 33,75^{\circ} ; 45^{\circ} ; 56,25^{\circ} ; 67,5^{\circ} ; 78,75^{\circ} ; 90^{\circ}$.

Погрешность $\Delta \mathrm{Z}_{\mathrm{DR}}$ в дБ определялась по следующей формуле: 


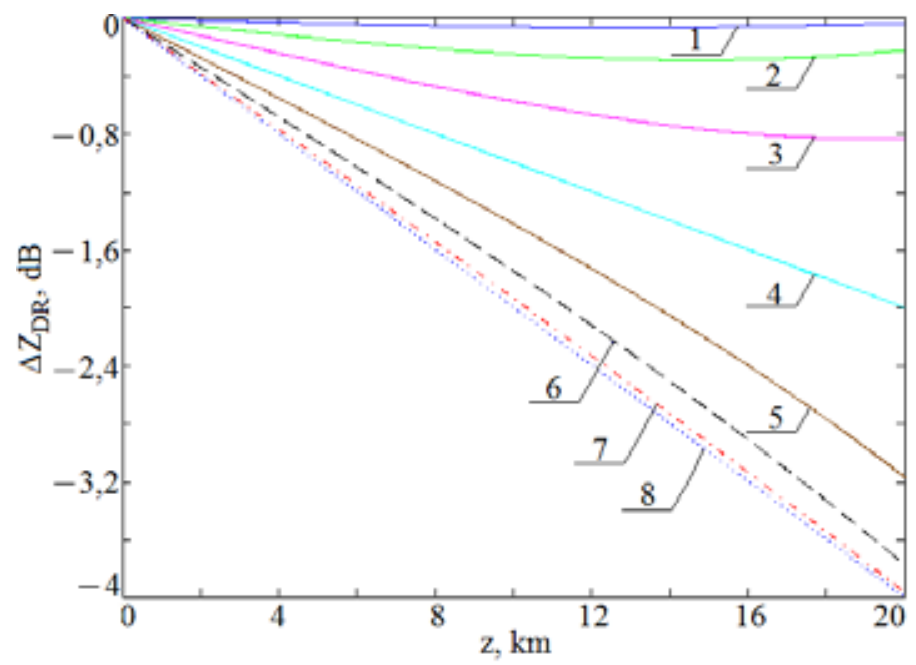

Рис. 5. $\Delta \mathrm{Z}_{\mathrm{DR}}$ при $\mathrm{R}=50$ мм/ч при значениях $\beta_{\mathrm{Hi}}: 1-11,25^{\circ} ; 2-22,5^{\circ} ; 3-33,75^{\circ} ; 4-45^{\circ} ; 5-56,25^{\circ} ; 6-67,5^{\circ}$; $7-78,75^{\circ} ; 8-90^{\circ}$

Fig. 5. Differential radar reflectivity measurement error $\Delta \mathrm{Z}_{\mathrm{DR}}$ for the rain intensity $\mathrm{R}=50 \mathrm{~mm} / \mathrm{h}$ and polarization basis orientation angle $\beta_{\mathrm{Hi}}$ (in degrees): $1-0 ; 2-11,25 ; 3-22,5 ; 4-33,75 ; 5-45 ; 6-56,25 ; 7-67,5 ; 8-78,75$; $9-90$

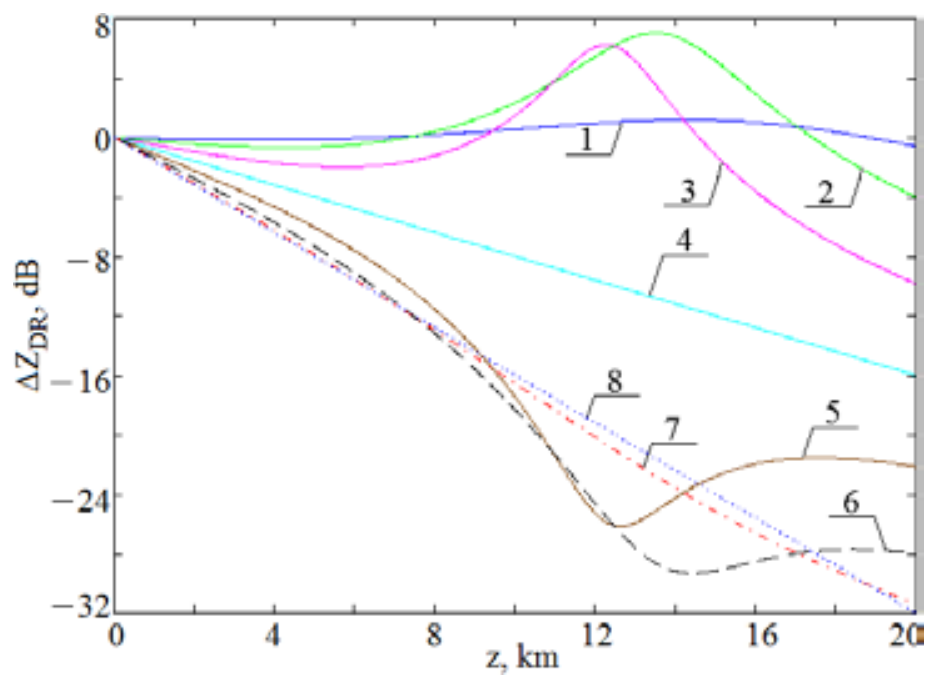

Рис. 6. $\Delta \mathrm{Z}_{\mathrm{DR}}$ при $\mathrm{R}=150$ мм/ч при значениях $\beta_{\mathrm{Hi}}: 1-11,25^{\circ} ; 2-22,5^{\circ} ; 3-33,75^{\circ} ; 4-45^{\circ} ; 5-56,25^{\circ} ; 6-67,5^{\circ}$; $7-78,75^{\circ} ; 8-90^{\circ}$

Fig. 6. Differential radar reflectivity measurement error $\Delta \mathrm{Z}_{\mathrm{DR}}$ for the rain intensity $\mathrm{R}=150 \mathrm{~mm} / \mathrm{h}$ and polarization basis orientation angle $\beta_{\mathrm{Hi}}$ (in degrees): $1-0 ; 2-11,25 ; 3-22,5 ; 4-33,75 ; 5-45 ; 6-56,25 ; 7-67,5 ; 8-$ 78,$75 ; 9-90$

$$
\Delta \mathrm{Z}_{\mathrm{DR}}\left(\Delta \alpha, \Delta \Phi, \beta_{\mathrm{H}}\right)=\mathrm{Z}_{\mathrm{DR}}\left(\Delta \alpha, \Delta \Phi, \beta_{\mathrm{H}}=0\right)-\mathrm{Z}_{\mathrm{DR}}\left(\Delta \alpha, \Delta \Phi, \beta_{\mathrm{Hi}}\right),
$$

где $\beta_{\mathrm{Hi}}$ при $\mathrm{i}=1 \div 8$ (номер і соответствует номерам кривых на рис. 4-6) принимает значения 1 $11,25^{\circ} ; 2-22,5^{\circ} ; 3-33,75^{\circ} ; 4-45^{\circ} ; 5-56,25^{\circ} ; 6-67,5^{\circ} ; 7-78,75^{\circ} ; 8-90^{\circ}$ соответственно.

Результаты расчетов погрешности определения ДРЛО приведены на рис. 4-6. 


\section{Анализ результатов}

На рис. 1 и 2 видно, что на трассах длиной до 10 км имеет место линейная зависимость ДРЛО от длины трассы z. В случае осадков с интенсивностью 150 мм/ч зависимость $Z_{\mathrm{DR}}(\mathrm{z})$ (1) имеет наиболее ярко выраженный характер (рис. 3). При этом линейный участок зависимости имеет место лишь до дальности 4 км. На трассах длиной свыше 12 км появляются экстремумы при $\beta_{\mathrm{H}}=22,5^{\circ} ; 33,75^{\circ} ; 56,25^{\circ} ; 67,5^{\circ}$. Эти экстремумы для указанных углов достигают значений $16\left(\beta_{\mathrm{H}}=33,75^{\circ} ; 56,25^{\circ}\right)$ и 18 дБ $\left(\beta_{\mathrm{H}}=22,5^{\circ} ; 67,5^{\circ}\right)$ по абсолютной величине соответственно.

Из приведенных на рис. 4-6 расчетных значений погрешности видно, что с увеличением длины трассы для осадков с интенсивностью 12,5 и 50 мм/ч зависимость $\Delta Z_{\mathrm{DR}}(\mathrm{z})$ остается практически линейной. При этом максимальные значения $\Delta Z_{\mathrm{DR}}(\mathrm{z})$ достигаются при $\beta_{\mathrm{H}}=90^{\circ}$ и составляют величины - 0,8 и - 4 дБ для интенсивности осадков 12,5 и 50 мм/ч соответственно.

В случае осадков с $\mathrm{R}=150$ мм/ч при углах $\beta_{\mathrm{H}}$, превышающих значение $22,5^{\circ}$, появляется нелинейность изменений $\Delta \mathrm{Z}_{\mathrm{DR}}(\mathrm{z})$ при длине трассы, превышающей 4 км.

\section{Заключение}

Получены расчетные соотношения для оценки ДРЛО и погрешностей этой оценки, имеющих место при дистанционном зондировании среды, заполненной гидрометеорами. Изменчивость величины ДРЛО имеет выраженный линейный характер (для различных углов ориентации собственного базиса среды распространения относительно измерительного базиса) только для дождей с интенсивностями $\mathrm{R}=12,5$ и 50 мм/ч.

Изменчивость $Z_{\mathrm{DR}}(\mathrm{z})$ имеет ярко выраженный характер при интенсивности осадков $\mathrm{R}=150$ мм/ч. На дальностях 12-14 км появляются экстремумы. При этом, как видно на рис. 6, имеют место весьма значительные величины погрешностей оценки $\mathrm{Z}_{\mathrm{DR}}(\mathrm{z})$.

Характерной особенностью изменчивости $Z_{\mathrm{DR}}(\mathrm{z})$ при углах $\beta_{\mathrm{H}}$ больше 45 градусов выступает появление положительных значений указанной величины, что может служить дополнительным информационным признаком наличия существенного изменения ориентации собственного базиса метеообъекта относительно измерительного, обусловленного, например, поперечным сдвигом ветра на трассе распространения. Кроме того, появление аномально высоких значений $Z_{\mathrm{DR}}(\mathrm{z})$ (6 дБ и выше) на участках трассы относительно небольшой протяженности свидетельствует о наличии осадков с высокой интенсивностью на данном участке трассы.

К числу перспектив дальнейших исследований по данной теме нужно отнести, прежде всего, поиск алгоритмов функционирования поляризационных радиолокаторов, позволяющих исключить воздействие ориентации собственного базиса метеообразования на оценку измеряемых поляризационных параметров.

\section{Список литературы}

[1] Вовшин Б.М., Вылегжанин И.С., Жуков В.Ю., Пушков А.А., Щукин Г.Г. Теория и практика поляризационных измерений в метеорологической радиолокации. Вторые Всероссийские Аpмандовские чтения. Муром: МИВлГУ, 2012, 1, 49-54. [Vovshin B.M., Vilegzhanin I.S., Zhukov V.U., Pushkov A.A., Shukin G.G. Theory and practice of polarization measurments in meteorological radar surveillance. The Second All-Russian Armand readings, Murom, 2012, 1, 49-54 (in Russian)]. 
[2] Zahrai A., Zrnic D.S. The 10-cm-Wavelength Polarimetric Weather Radar at NOAA's National Severe Storms Laboratory. Journal of atmospheric and oceanic technology, 1993, 10(5), 649-662.

[3] Zrnic D.S., Ryzkov A.V. Polarimetry for Weather Surveillance Radars. Bulletin of the American Meteorological Society, 1999, 80(3), 389-406.

[4] Melnikov V.M. Statistics of the polarimetric variables estimated in the AHV and AHV modes. Journal of applied meteorology and climatology, 2011, 50, 859-872.

[5] Масалов Е.В., Татаринов В.Н. Поляризационные измерения в задачах радиолокационной метеорологии. Зарубежная радиоэлектроника, 1987, 4, 44-52. [Masalov E.V., Tatarinov V.N. Polarization measurements in radar meteorology. Telecommunications and Radio Engineering, 1987, 4, 44-52 (in Russian)].

[6] Масалов Е.В. Трансформация линейно поляризованных электромагнитных волн в средах, содержащих гидрометеоры. Актуальные проблемы электронного приборостроения, Новосибирск: НГТУ, 2010, 2, 77-79. [Masalov E.V. Transformation of the linearly polarized electromagnetic waves in mediums, filled with hydrometeors. Engineering proceedings of 10th international conference on actual problems of electronic instrument, Novosibirsk, 2010, 2, $77-79$ (in Russian)].

[7] Огути Т. Распространение и рассеяние электромагнитных волн в дожде и других гидрометеорах. ТИИЭР, 1983, 71(9), 6-65. [Oguti Т. Propagation and dissipation of electromagnetic waves in rain and other hydrometeors. Proc. Of IEEE, 1983, 71(9), 6-65].

[8] Родимов А. П., Поповский В.В., Дмитриев В.И. Особенности использования поляризационных параметров электромагнитных волн в системах связи миллиметрового диапазона. Зарубежная радиоэлектроника, 1980, 7, 25-37. [Rodimov A.P., Popovsky V.V., Dmitriev V.I. The features of using electromagnetic waves polarization parameters in the millimeter wavelength range communication systems. Telecommunications and Radio Engineering, 1980, 7, 25-37 (in Russian)]. 